TITLE:

\title{
Light-emission properties of quantum dots embedded in a photonic double-heterostructure nanocavity
}

\section{$\operatorname{AUTHOR}(\mathrm{S})$ :}

Stumpf, WC; Fujita, M; Yamaguchi, M; Asano, T; Noda, S

\section{CITATION:}

Stumpf, WC ...[et al]. Light-emission properties of quantum dots embedded in a photonic double-heterostructure nanocavity. APPLIED PHYSICS LETTERS 2007, 90 (23): 231101.

\section{ISSUE DATE:}

2007-06-04

URL:

http://hdl.handle.net/2433/50063

\section{RIGHT:}

Copyright 2007 American Institute of Physics. This article may be downloaded for personal use only. Any other use requires prior permission of the author and the American Institute of Physics. 


\title{
Light-emission properties of quantum dots embedded in a photonic double-heterostructure nanocavity
}

\author{
Wolfgang C. Stumpf, ${ }^{a)}$ Masayuki Fujita, Makoto Yamaguchi, \\ Takashi Asano, and Susumu Noda ${ }^{\text {b) }}$ \\ Department of Electronic Science and Engineering, Kyoto University, Kyoto-Daigaku-Katsura, Nishikyo-Ku, \\ Kyoto 615-8510, Japan
}

(Received 27 December 2006; accepted 9 May 2007; published online 4 June 2007)

\begin{abstract}
The authors report on the investigation of the light-emission properties of quantum dots in a photonic double-heterostructure nanocavity. The emission spectrum clearly allows the identification of the cavity and waveguide band edge modes. The frequency and polarization characteristics are in good agreement with three-dimensional finite-difference time-domain calculations. Resonant waveguide band edge mode excitation is demonstrated to reduce the background intensity that does not originate from the cavity and consequently cavity mode properties become evident. Finally, the $Q$ value is estimated and discussed. (C) 2007 American Institute of Physics.
\end{abstract}

[DOI: $10.1063 / 1.2746059]$

In order to develop and improve photonic light sources such as ultrahigh efficient emitters ${ }^{1}$ and quantuminformation-processing ${ }^{2}$ devices, one has to understand the fundamental phenomena of the underlying cavity quantum electrodynamics ${ }^{3}$ (weak and strong couplings, enhancement and suppression of spontaneous emission, quantum entanglement, decoherence, etc.). Nanostructured materials that can be tailored to the desired quantum properties allow researchers to probe these phenomena and have hence stirred up much attention during the past year. ${ }^{4}$ Moreover, the materials chosen make prospective optoelectronic integrated circuits more feasible.

Quantum dots (QDs) are nanostructures that are attractive as photon emitters for the objectives mentioned above. The electronic states in QDs are determined by three dimensional (3D) carrier confinement which yields a deltalike density of states. ${ }^{5}$ Spontaneous emission ${ }^{6}$ arising from QDs ${ }^{7}$ can be controlled by a photonic band gap (PBG) in a photonic crystal (PC) where the dielectric constant varies on the order of a photon's wavelength, consequently inhibiting certain photon energies and direction ranges from propagating. ${ }^{8}$ Hence for developing the aforementioned photon sources, the combination of QDs and PCs is most promising because both carriers and photons can be manipulated. Intentional defects in a PC lattice such as a missing line [waveguide (WG)] or single points (cavity) allow guiding and confinement of photons in a volume of wavelength order. The efficiency of photon localization of PC NCs can be described by $Q / V$, where $Q$ and $V$ are the cavity's quality factor and modal volume, respectively. An ultrahigh $Q$ with $V$ of about one cubic wavelength in the material can be achieved using the double-heterostructure (DH) nanocavity (NC) design. In fabricating passive components, theoretical and experimental $Q$ factors that exceeded 600000 have been reported in silicon, ${ }^{9}$ similar devices have been demonstrated in GaAs as well. ${ }^{10,11}$ However, the interesting combination of QDs and a DH NC, exemplarily shown in Fig. 1(a), has not been dem-

\footnotetext{
${ }^{a)}$ FAX:

+81-75-383-2320;

electronic

mail:

wolfgang@qoe.kuee.kyoto-u.ac.jp

b)Electronic mail: snoda@kuee.kyoto-u.ac.jp
}

onstrated yet. In this study the light-emission phenomena of QDs embedded in a DH NC are investigated.

Figure 1(b) schematically shows the cross section of the substrate which was grown by molecular beam epitaxy on an epitaxy-ready semi-insulating (100) GaAs substrate. First a $200 \mathrm{~nm}$ GaAs buffer layer, then an $\mathrm{Al}_{0.7} \mathrm{Ga}_{0.3} \mathrm{As}$ sacrificial layer of $1.2 \mu \mathrm{m}$ thickness was epitaxially grown, and followed by a $200 \mathrm{~nm}$ GaAs PC slab layer that incorporated a QD layer at its vertical center plane. After the first $100 \mathrm{~nm}$ growth of the slab layer, 2.6 at. ML of InAs were supplied at $520{ }^{\circ} \mathrm{C}$ substrate temperature and low arsenic pressure of about $5.6 \times 10^{-7} \mathrm{mbar}$ in order to form self-organized QDs in the Stranski-Krastanow mode. ${ }^{12}$ The growth rate was $0.033 \mathrm{ML} / \mathrm{s}$. The capping layer, i.e., the last $100 \mathrm{~nm}$ of the slab layer, was grown at $480{ }^{\circ} \mathrm{C}$ to avoid InAs/GaAs intermixing. Under similar conditions, another substrate was fabricated $^{13}$ without the capping layer to determine the QD spatial properties. The average lateral size was $30 \mathrm{~nm}$, the height was between 4 and $8 \mathrm{~nm}$, and with a density of about $4 \times 10^{10} \mathrm{~cm}^{-2}$ found by atomic force microscopy (AFM) imaging shown in Fig. 1(c).

The PCs were defined by a pattern written by electronbeam lithography on a resist layer, transferred onto the sub-

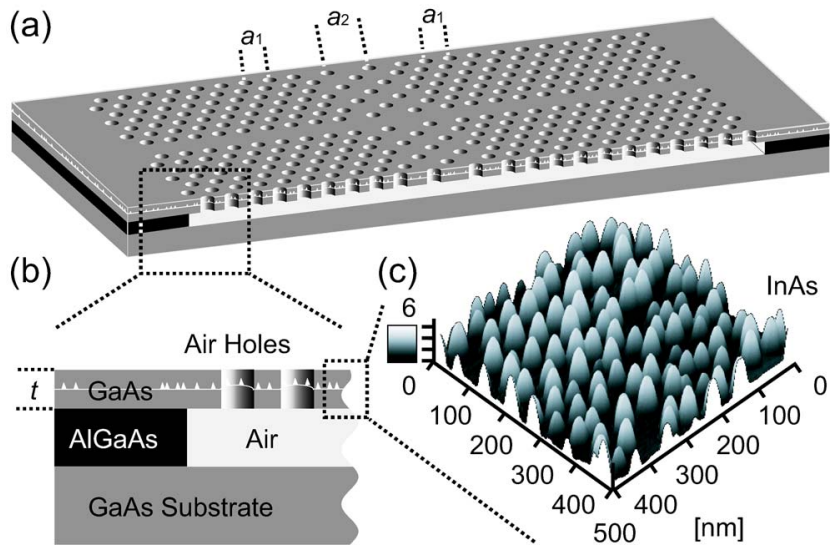

FIG. 1. (a) Two different lattice constants $\left(a_{1}, a_{2}\right)$ along the W1 WG correspond to the $\mathrm{DH} \mathrm{NC}$ where (b) magnifies the substrate cross section and (c) shows an AFM image of the QDs in nanometer scale. 


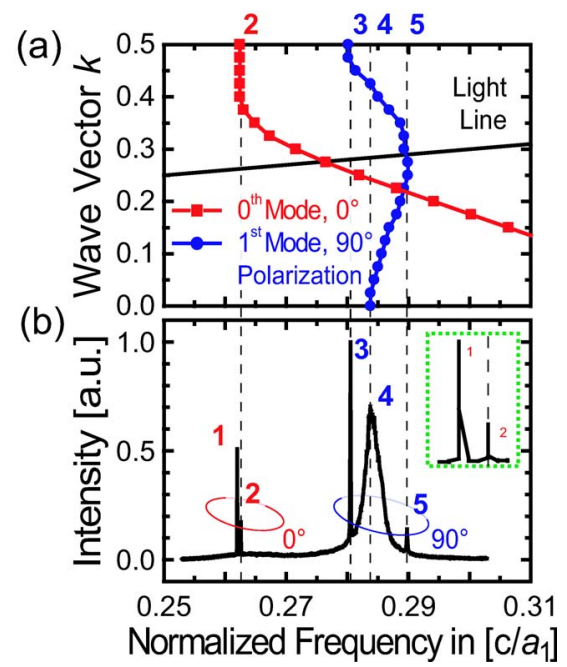

FIG. 2. (Color online) (a) Dispersion relation of the W1 WG modes obtained by 3D FDTD and (b) an example for the experimental PL spectrum of the QDs, with the modes grouped by polarization. In the inset on the right, modes 1 and 2 are expanded. The excitation power density is $450 \mathrm{~W} / \mathrm{cm}^{2}$.

strate by HI/Xe inductively coupled plasma etching, ${ }^{14}$ and later undercut by removing the sacrificial layer using $\mathrm{HCl}-$ based wet etching. ${ }^{15}$ As a result the fabricated symmetric air-slab-air structures confine light in plane by a twodimensional (2D) PBG and out of plane by total internal reflection. It should be noted that the finished structure shown in Fig. 1(a) represents only one of the DH NC designs that can obtain an ultrahigh $Q$ value.

The presented design of this $\mathrm{DH} \mathrm{NC}$ basically consists of a 2D PC slab (thickness $t=0.7 a_{1}$ ), where a missing row of air holes (radius $r=0.3 a_{1}$ ) in the $\Gamma-J$ direction of the triangular lattice structure acts as a line defect (W1) WG. ${ }^{16}$ At the PC WG center, the lattice constant $a_{1}=300 \mathrm{~nm}$ is expanded to $a_{2}=308 \mathrm{~nm}$ in the WG direction for two periods while it remains unchanged in the lateral direction for lattice matching. Thus a PC waveguide $\mathrm{NC}$ is formed due to the photonic mode-gap effect. ${ }^{9}$

For low temperature characterization, samples are investigated in a liquid-helium flow cryostat operating at $3 \mathrm{~K}$ with optical access. Continuous wave (cw) light from either a diode laser (wavelength: $\lambda_{\mathrm{exc}}=830 \mathrm{~nm}$ ) or tunable Ti:sapphire (Ti:Sa) laser $\left(\lambda_{\text {exc }}=1000-1100 \mathrm{~nm}\right)$ is focused by a $20 \times$ microscope objective (numerical aperture $=0.4$ ) onto the top of the substrate for optical excitation. Emitted light is collected by the same microscope objective and fiber coupled to a $0.22 \mathrm{~nm}$ resolution spectrometer (length $l=0.35 \mathrm{~m}$ ) with a liquid nitrogen cooled InGaAs detector array. For $Q$ measurements a similar type of spectrometer $(l=0.75 \mathrm{~m})$ was used.

Figure 2(a) shows the W1 WG modes calculated by 3D finite-difference time-domain (FDTD) method. In the FDTD calculation, a wavelength dependence of the refractive index was taken into account. The two groups of photoluminescence (PL) peaks $\{1,2\}$ and $\{3,4,5\}$ were given refractive indices of 3.34 and 3.38, respectively, in order to fit the experimental data to the WG band edges. ${ }^{17}$ According to the W1 WG modes' electric field distribution, ${ }^{18}$ the rectangles indicate the zeroth order (even) and the circles the first order (odd) W1 WG mode. The modes' polarizations were calculated and found to be $0^{\circ}$ for the even and $90^{\circ}$ for the odd W1 WG mode, where $0^{\circ}$ is defined as perpendicular and $90^{\circ}$ as Downloaded 05 Mar 2008 to 130.54.110.22. Redistribution subjec

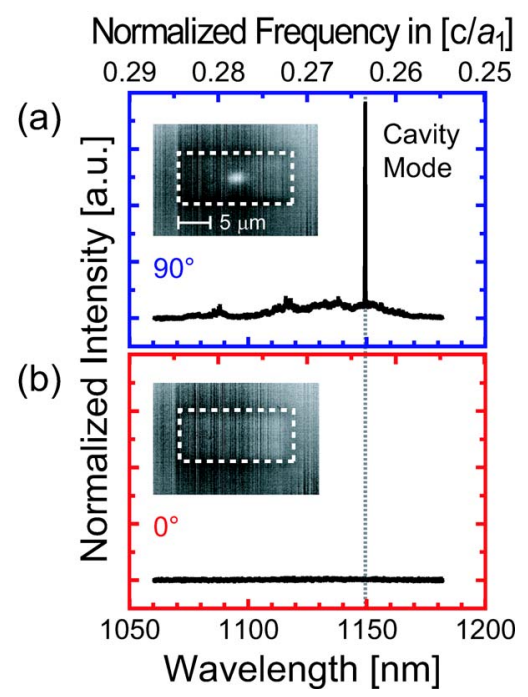

FIG. 3. (Color online) Excitation conditions on resonance. The excitation wavelength for both spectra is $\lambda_{\text {exc }}=\lambda_{4}=1054 \mathrm{~nm}$ (compare Fig. 2). In (a) the excitation matches the mode 4 polarization, whereas in (b) it is perpendicular. The insets show IR images. The localized emission from the NC can only be observed if the excitation laser light matches in wavelength and polarization. The excitation power densities are $90 \mathrm{~W} / \mathrm{cm}^{2}$ for (a) and $150 \mathrm{~W} / \mathrm{cm}^{2}$ for $(\mathrm{b})$.

parallel to the WG. In Fig. 2(b) a representative PL spectrum at $3 \mathrm{~K}$ is shown for wetting layer (WL) excitation at $\lambda_{\text {exc }}$ $=830 \mathrm{~nm}$. For the WL excitation, the energy of the excited carriers is sufficient to recombine in an arbitrary QD, giving rise to a broad background PL, allowing the study of all apparent modes of the sample. As expected from 3D FDTD results, peak 1 in the PL spectrum was experimentally corroborated in wavelength and polarization to be the $\mathrm{NC}$ mode. Mapping measurements demonstrated that the $\mathrm{NC}$ mode (peak 1) could only be detected at the PC NC center whereas the WG mode (peak 2) appears exclusively as scattered light at the PC WG exit facets. The low group velocity band edges of the dispersion relation in Fig. 2(a) were also found to be in agreement with the wavelength and polarization properties of PL peaks 2-5 of Fig. 2(b). Those properties were calculated and confirmed separately for the NC mode peak 1 .

In the PL spectrum in Fig. 2(b), peak 4 has a notably broad linewidth [full width at half maximum (FWHM) is $\sim 12 \mathrm{~nm}$ ] compared to the other modes (FWHMs of peak 2 and 5 are $\sim 0.6 \mathrm{~nm}$, peak 3 is $\sim 0.4 \mathrm{~nm}$ ). Only peak 4 , which originates from the $\Gamma$ point of the PC lattice and is a PBGguided mode ${ }^{19}$ lies above the light line and hence is a leaky mode. To confirm the effect of the light line, each mode's $Q$ factor was calculated by 3D FDTD and found to be in good agreement with the experiment. Light from outside can easily couple to a leaky mode, thus peak 4 facilitates resonant excitation which is thought to reduce the excited area to the WG alone and reduce the background intensity.

Basically there are two requirements for the resonant excitation: First, the $90^{\circ}$ polarization has to be matched. Figure 3 illustrates $90^{\circ}$ (a) and $0^{\circ}$ (b) polarizations of the excitation laser, for the latter only background PL is detected because the WG cannot be excited. The second requirement is to tune the excitation laser $\lambda_{\text {exc }}$ to the wavelength of peak 4 , where $\lambda_{4}=1054 \mathrm{~nm}$. By adjusting the $90^{\circ}$ polarized $\mathrm{cw}$ Ti:Sa excitation laser wavelength to resonate with the WG band edge mode wavelength, the energy transfer of the driving excitation laser field into the $\mathrm{NC}$ mode is maximized. to AlP license or copyright; see http://apl.aip.org/apl/copyright.jsp 


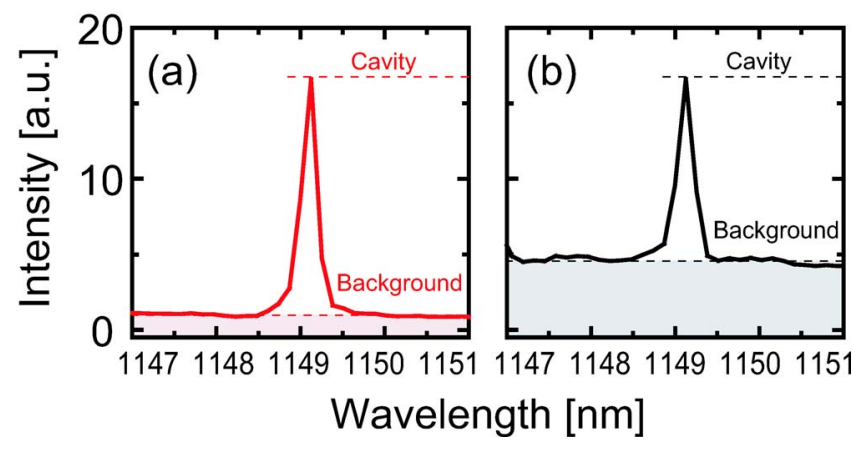

FIG. 4. (Color online) Spectra for (a) resonant and (b) nonresonant excitations are compared. The excitation power densities are $90 \mathrm{~W} / \mathrm{cm}^{2}$ for (a) and $80 \mathrm{~W} / \mathrm{cm}^{2}$ for (b).

The on/off resonance emission intensity ratio is $I_{\text {on }} / I_{\text {off }}=10$ for such a photoluminescence excitation measurement when the probed NC (peak 1) wavelength is $\lambda_{1}=1148 \mathrm{~nm}$. The resulting curve corresponds to the shape of the excited peak 4. The resonant case, where both of the conditions for excitation laser light are matched, viz., $\lambda_{\text {exc }}=\lambda_{4}=1054 \mathrm{~nm}$ and $90^{\circ}$ polarization, is displayed in Fig. 3(a). Here the resonant excitation can be recognized from the appearance of the $\mathrm{NC}$ mode in the spectrum and localized emission at the NC area, shown in the inset taken by an infrared (IR) camera. In Fig. 3(b) the requirements are not met, thus the NC is not excited with either IR emission imaging or detectable mode peaked spectrum.

Figure 4 shows the spectra for resonant excitation and nonresonant WL excitation. The ratio of NC mode to background intensity is improved by a factor of 6 for resonant excitation. Assuming a typical focus spot to be $5 \mu \mathrm{m}$ in diameter and centered at the $\mathrm{DH} \mathrm{NC}$, the total area is reduced by the PC air holes $1-2 \pi r^{2} / \sqrt{3} \cong 0.7$ and contains $N$ QDs. The WG itself has only $N_{\mathrm{W} 1}(<N)$ dots that are resonantly excited. The ratio $N / N_{\mathrm{W} 1}$ is about 8 , which is close to the experimental result. Depending on the focal spot size, this figure of merit can be changed by using a different focus size, thus being limited by the collection efficiency of the optical detection system.

In resonant excitation, PL spectra taken with a spectrometer with $0.025 \mathrm{~nm}$ resolution permitted the estimation of the NC $Q$, as shown in Fig. 5. The NC linewidth has a FWHM of $0.061 \mathrm{~nm}$ that yields a $Q$ around 19000 , although the passive $Q_{\text {design }}$ is larger than 5000000 . That means that the NC's experimental $Q$ factor is largely determined by various scattering and absorption components. ${ }^{20}$ Additionally, the authors think that the influence of the excitons cannot be entirely excluded since photons emitted from the NC might be supplied from the excitons by some kind of mutual interaction. We note that the exciton linewidth is about $0.051 \mathrm{~nm}$ when out of resonance with the NC mode.

In conclusion the combination of a DH NC with QDs was demonstrated. The emission characteristics were studied and the findings indicate good agreement with 3D FDTD results for the frequency and polarization properties. The

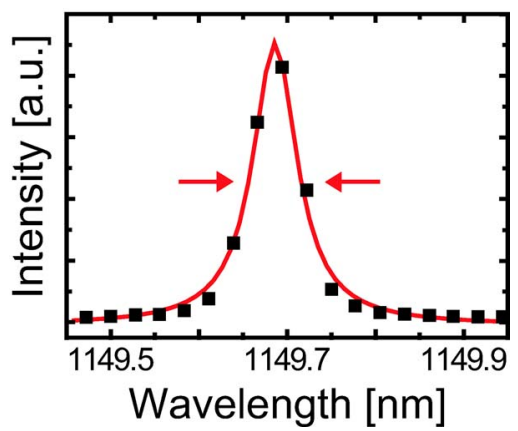

FIG. 5. (Color online) Scatter shows the raw data fitted by a Lorentzian function to estimate the experimental NC $Q$. FWHM is $0.061 \mathrm{~nm}$.

resonant excitation technique was applied where the NC excitation could be recognized by localized NC emission. This technique allowed for the estimation of the NC $Q$, within certain limits. The results are encouraging for defining future experiments.

The authors would like to thank Yoshinori Tanaka for helpful discussions. They gratefully acknowledge support by the Japan Science and Technology Agency (CREST), an IT project, and a grant-in-aid of the Ministry of Education, Culture, Sports, Science and Technology of Japan.

${ }^{1}$ S. Noda, Science 314, 260 (2006).

${ }^{2}$ D. Loss and D. P. DiVincenzo, Phys. Rev. A 57, 120 (1998).

${ }^{3}$ G. Khitrova, H. M. Gibbs, M. Kira, S. W. Koch, and A. Scherer, Nat. Phys. 2, 81 (2006).

${ }^{4}$ P. Bhattacharya, S. Ghosh, and A. D. Stiff-Roberts, Annu. Rev. Mater. Res. 34, 1 (2004).

${ }^{5}$ Y. Arakawa and H. Sakaki, Appl. Phys. Lett. 40, 939 (1982).

${ }^{6}$ M. Fujita, S. Takahashi, Y. Tanaka, T. Asano, and S. Noda, Science 308, 1296 (2005)

${ }^{7}$ D. Englund, D. Fattal, E. Waks, G. Solomon, B. Zhang, T. Nakaoka, Y. Arakawa, Y. Yamamoto, and J. Vučković, Phys. Rev. Lett. 95, 013904 (2005).

${ }^{8}$ E. Yablonovitch, Phys. Rev. Lett. 58, 2059 (1987).

${ }^{9}$ B.-S. Song, S. Noda, T. Asano, and Y. Akahane, Nat. Mater. 4, 207 (2005).

${ }^{10}$ R. Herrmann, T. Sünner, T. Hein, A. Löffler, M. Kamp, and A. Forchel, Opt. Lett. 31, 1229 (2006).

${ }^{11}$ E. Weidner, S. Combrié, N.-V.-Q. Tran, A. De Rossi, J. Nagle, S. Cassette, A. Talneau, and H. Benisty, Appl. Phys. Lett. 89, 221104 (2006).

${ }^{12}$ I. N. Stranski and L. Krastanow, Sitzungsber. Akad. Wiss. Wien, Math.Naturwiss. Kl., Abt. 2B 146, 797 (1937).

${ }^{13}$ K. Kounoike, M. Yamaguchi, M. Fujita, T. Asano, J. Nakanishi, and S. Noda, Electron. Lett. 41, 1402 (2005).

${ }^{14}$ M. Fujita, A. Sugitatsu, T. Uesugi, and S. Noda, Jpn. J. Appl. Phys., Part 2 43, L1400 (2004)

${ }^{15}$ M. Fujita, S. Takahashi, T. Asano, Y. Tanaka, K. Kounoike, M. Yamaguchi, J. Nakanishi, W. Stumpf, and S. Noda, J. Opt. A, Pure Appl. Opt. 8, S131 (2006).

${ }^{16}$ A. Chutinan and S. Noda, Phys. Rev. B 62, 4488 (2000).

${ }^{17}$ S. Gehrsitz, F. K. Reinhart, C. Gourgon, N. Herres, A. Vonlanthen, and H. Sigg, J. Appl. Phys. 87, 7825 (2000).

${ }^{18}$ S.-H. Kim, G.-H. Kim, S.-K. Kim, H.-G. Park, Y.-H. Lee, and S.-B. Kim, J. Appl. Phys. 95, 411 (2004).

${ }^{19}$ K. Kiyota, T. Kise, N. Yokouchi, T. Ide, and T. Baba, Appl. Phys. Lett. 88, 201904 (2006).

${ }^{20}$ T. Asano, B.-S. Song, and S. Noda, Opt. Express 14, 1996 (2006). 